\title{
Whole-grain consumption and the risk of all-cause, CVD and cancer mortality: a meta-analysis of prospective cohort studies
}

\author{
Honglei Wei ${ }^{1,2} \dagger$, Zong $\mathrm{GaO}^{3} \dagger$, Rui Liang ${ }^{1}$, Zengqiang $\mathrm{Li}^{1}$, Hong $\mathrm{HaO}^{4}$ and $\mathrm{Xu} \mathrm{Liu}^{1 *}$ \\ ${ }^{1}$ Department of Sports, Jiaotong University, Chengdu, Sichuan Province, 611756, People's Republic of China \\ ${ }^{2}$ School of Economics and Management, Southwest Jiaotong University, Chengdu, Sichuan Province, 611756, People's \\ Republic of China \\ ${ }^{3}$ Department of Neurosurgery, the Affiliated Hospital of Shandong Traditional Chinese Medicine University, Jinan, Shandong \\ Province, 250011, People's Republic of China \\ ${ }^{4}$ School of Mathematics, Southwest Jiaotong University, Chengdu, Sichuan Province, 611756, People's Republic of China \\ (Submitted 29 October 2015 - Final revision received 18 March 2016 - Accepted 8 April 2016 - First published online 24 May 2016)
}

\section{Abstract}

Results of the relationships between dietary whole-grain consumption and the risk of all-cause, CVD and cancer-specific mortality are mixed. We summarised the evidence based on a meta-analysis of prospective cohort studies. Pertinent studies were identified by searching articles in the MEDLINE and EMBASE databases up to 20 January 2016 and by reviewing the reference lists of the retrieved articles. Random-effects models were used to calculate summary relative risks (SRR) and 95\% CI. In all, eleven prospective studies (ten publications) were included in the meta-analysis. There were a total of 816599 subjects and 89251 cases of all-cause mortality. On the basis of the highest $v$. the lowest categories of intake, whole grains may be associated with a lower risk of mortality from all causes (SRR 0.87; 95\% CI 0.84, 0.90), CVD (SRR 0.81; 95\% CI 0.75, 0.89) and all cancers (SRR 0.89; $95 \%$ CI $0.82,0.96$ ). For each 3 servings/d increase in whole-grain intake, there was a $19 \%$ reduction in the risk of all-cause mortality (SRR $0.81 ; 95 \%$ CI 0.76, 0.85), a $26 \%$ reduction in CVD mortality (SRR 0.74; 95\% CI 0.66, 0.83) and a 9\% reduction in cancer mortality (SRR 0.91; 95\% CI 0.84, 0.98). The current meta-analysis provides some evidence that high intake of whole grains was inversely associated with the risk of all-cause, CVD and cancer-specific mortality. Further well-designed studies, including clinical trials and in different populations, are required to confirm our findings.

\section{Key words: Whole grains: All-cause mortality: CVD: Meta-analyses}

Many dietary guidelines have recommended consumption of whole-grain foods by the general population ${ }^{(1)}$. Whole grains consist of three botanically defined parts: bran, germ and endosperm. The outer bran and germ contain various nutrients, including fibre, several vitamins and minerals and phytochemicals. Human feeding trials and mechanistic research have suggested that whole-grain foods and its constituents have beneficial effects on glucose metabolism, blood lipid profiles, endothelial function and inflammation ${ }^{(2,3)}$. In addition, an inverse association between consumption of whole grain and the risk of obesity ${ }^{(4,5)}$, type 2 diabetes mellitus $(\mathrm{DM})^{(6,7)}, \mathrm{CVD}^{(8)}$ and certain cancers ${ }^{(8,9)}$ has been found in a number of large prospective studies.

Of chronic non-communicable diseases, cancer and CVD are the main causes of death ${ }^{(10)}$. Worldwide, chronic noncommunicable diseases caused two out of every three deaths in 2010, increasing by approximately eight million between 1990 and $2010^{(10)}$. Given the reduction in risk of the main chronic non-communicable observed in whole-grain eaters, we hypothesised that whole-grain intake might be associated with a reduced risk of all-cause mortality. A number of epidemiological studies have investigated the relationship between consumption of whole grains and risk of all-cause mortality ${ }^{(11-20)}$. Some have found an inverse relationship between whole-grain intake and risk of all-cause mortality ${ }^{(11,13-16)}$; others have found no significant associations ${ }^{(17)}$. To our knowledge, no metaanalyses have looked at the effects of whole-grain consumption on the risk of all-cause, CVD and cancer mortality. We therefore undertook a meta-analysis of prospective cohort studies exploring these associations.

\section{Methods \\ Literature search}

The meta-analysis was undertaken in accordance with the preferred reporting items for systematic reviews and meta-analysis guidelines ${ }^{(21)}$. Two investigators (H. W. and Z. G.) systematically searched MEDLINE and EMBASE from their inception through 20 January 2016 in order to identify all

Abbreviations: DM, diabetes mellitus; RR, relative risk; SRR, summary relative risk.

* Corresponding author: Professor X. Liu, fax +860286636 7449, email liu611756@126.com

$\dagger$ Both authors contributed equally to this work. 
published articles on this topic. The search strategy included the following medical subject headings or key words: (1) cereals OR grains OR wheat OR rice OR bread; (2) fatal OR death OR mortality; (3) prospective OR cohort. The search was limited to studies in English. References from all relevant articles were hand-searched to identify additional relevant studies.

\section{Study selection}

The same two reviewers (H. W. and Z. G.) independently analysed citations at the levels of title, abstract and full text. To be included, studies had to meet the following criteria: (1) prospective cohort study design; (2) whole grains as the exposure of interest with all-cause mortality as the end point of interest; (3) relative risks (RR) and corresponding 95\% CI given for the association between whole-grain intake and the occurrence of total, CVD and cancer mortality. If the same population was the subject of more than one report, we included in our meta-analysis the one with the longest follow-up time. Studies on foods that were based on individual grain components (e.g. bran or germ) or specific types of grain (such as brown rice or oats) were excluded. Studies that examined the effects of high fibre, dietary fibre or cereal fibre, but in which the specific effect of whole grains could not be determined, were also excluded.

\section{Data extraction and study quality assessment}

We extracted the data using a standardised form. Publication characteristics were recorded as follows: first author's surname, publication year, study location, length of follow-up, number of participants, baseline mean age, methods of dietary assessment, number of events, confounding factors and RR estimates from the most fully adjusted model and the corresponding $95 \%$ CI. Two researchers (H. W. and Z. G.) independently extracted the data. Disagreements were resolved by discussion involving a third researcher (X. L.). We used the nine-star Newcastle-Ottawa Scale (NOS) to assess the risk of bias ${ }^{(26)}$; scores of seven or more were taken to indicate high-quality studies ${ }^{(26)}$.

\section{Statistical methods}

Adjusted RR and 95\% CI were combined and weighted to produce summary RR using a random-effects model. This was chosen, rather than a fixed-effects model, in order to account for anticipated heterogeneity between studies ${ }^{(22)}$. RR were plotted against their corresponding standard errors to produce funnel plots, which were checked for asymmetry in order to investigate publication bias using Begg's adjusted rank correlation test and Egger's linear regression test ${ }^{(23,24)}$. To estimate the effect of publication bias, we used the trim-and-fill computation ${ }^{(25)}$.

The extent of heterogeneity in pooled estimates was investigated using both the $Q$ statistic and the $I^{2}$. Results were classed as heterogeneous if $P<0 \cdot 10 . I^{2}$ is the part of the total variation that is explained by between-study variables. $I^{2}$ values of approximately 25,50 and $75 \%$ are considered to indicate low, moderate and high heterogeneity, respectively ${ }^{(26)}$.
To explore the sources of heterogeneity, subgroup and meta-regression analyses were performed according to sex, baseline mean age, duration of follow-up and methods of dietary assessment. Adjustments were made for confounding factors, including histories of hypertension, dyslipidaemia and type 2 DM. Further sensitivity analysis was performed to evaluate whether there was evidence of excessive influence from individual study by removing each individual study and assessing the resulting effect on estimates and measures of heterogeneity ${ }^{(27)}$.

The dose-response relationships in forest plots were assessed by 3 servings/d increments. We used the generalised least-squares trend estimation method developed by Greenland \& Longnecker $^{(28)}$ and Orsini \& Greenland ${ }^{(29)}$. This requires that the distribution of cases and person-years or non-cases and the RR with variance estimates for at least three quantitative exposure categories be known. When this information was not available, we estimated dose-response slopes using varianceweighted least-squares regression ${ }^{(28,29)}$. The median or mean level of whole-grain intake in each category was assigned to the corresponding RR for each study. If such values were not reported, we took the estimated midpoint between the upper and lower boundaries in each category as the average intake level. If the highest or lowest category was open-ended, we assumed the size of the open-ended interval to be the same as that of the adjacent interval. In studies that reported the intakes in grams per day, we used $30 \mathrm{~g}$ as a serving size for recalculation of the intakes to a common scale (servings) ${ }^{(27)}$. Potential non-linear dose-response relationships were examined using the best-fitting fractional polynomial models ${ }^{(30)}$, defined as those with the least deviance. A likelihood ratio test was used to determine the difference between non-linear and linear models to assess non-linearity ${ }^{(30)}$.

We used STATA, version 11.0 (StataCorp. LP) and R package, version 2.11.0 beta ( $\mathrm{R}$ Development Core Team) for the statistical analyses. A two-tailed $P<0.05$ was considered statistically significant.

\section{Results}

\section{Search results and study characteristics}

The search strategy yielded 802 articles, fifty-four of which had potential value and were available as full-text articles (Fig. 1). An additional three potential articles were found from scrutinising references. Following examination of the fifty-seven potential articles, forty-seven were excluded from the meta-analysis for a variety of reasons. The remaining ten articles were included in the meta-analysis. One article ${ }^{(14)}$ included two cohorts: the Nurses' Health Study and the Health Professionals Follow-Up Study. Consequently, eleven prospective cohort studies were analysed (Table 1).

These eleven studies included a total of 816599 subjects, ranging from 535 in the smallest cohort to 367442 in the largest cohort. The median duration of follow-up was 14 years (range: 5.5-26 years). There were 89251 cases of all-cause mortality (range: 165-46 067), 23280 CVD deaths (range: 89-11283) and 35189 cancer deaths (range: 169-19043). Eight studies were performed in the USA, one in Norway, one in Spain and one in Scandinavia. One study ${ }^{(16)}$ used a 3 -d food record as a tool 
Fig. 1. Flow diagram of the systematic literature search undertaken on whole-grain intake and the risk of all-cause and CVD mortality.

to collect dietary information, and the others used a validated FFQ. All studies provided RR that were adjusted for age, smoking, alcohol use, BMI and physical activity. Most studies provided RR that were adjusted for hypertension or measured blood pressure (seven studies), DM (five studies) and dyslipidaemia (four studies). According to the NOS, all studies were of high quality (online Supplementary Table S1).

\section{All-cause mortality}

High v. low analysis. In all, eleven studies from ten publications investigated the association between whole-grain intake and all-cause mortality risk. The summary relative risk (SRR) was 0.87 (95\% CI 0.84, 0.90) for subjects in the highest category of whole-grain consumption compared with those in the lowest category, with evidence of high heterogeneity among studies ( $P_{\text {heterogeneity }}<0.001 ; I^{2}=67 \cdot 3 \%$; Fig. 2(a)).

Dose-response analysis. In all, eight studies from seven publications were included in the dose-response analysis of wholegrain consumption and all-cause mortality risk (Fig. 2(b)). The SRR per 3 servings/d was 0.81 (95\% CI 0.76, 0.85), with evidence of heterogeneity $\left(I^{2}=79 \cdot 3 \%, P_{\text {heterogeneity }}<0.001\right)$.

\section{CVD mortality}

High v. low analysis. Nine studies from eight publications investigated the association between whole-grain intake and CVD mortality risk. The SRR was 0.81 (95\% CI $0.75,0.89)$ for subjects in the highest category of whole-grain consumption compared with those in the lowest category, with evidence of high heterogeneity among studies $\left(P_{\text {heterogeneity }}=0.013\right.$; $I^{2}=56.9 \%$; Fig. 3(a)).

Dose-response analysis. Eight studies from seven publications were included in the dose-response analysis of whole-grain consumption and CVD mortality risk (Fig. 3(b)). The SRR per 3 servings/d was 0.74 (95\% CI 0.66, 0.83), with evidence of high heterogeneity $\left(I^{2}=76 \cdot 3 \%, P_{\text {heterogeneity }}<0.001\right)$.

\section{Total cancer mortality}

High v. low analysis. Seven studies from six publications investigated the association between whole-grain intake and total cancer mortality risk. The SRR was 0.89 (95\% CI 0.82, 0.96) for subjects in the highest category of whole-grain consumption compared with those in the lowest category, with evidence of high heterogeneity $\left(P_{\text {heterogeneity }}=0 \cdot 007 ; I^{2}=63.6 \%\right.$; Fig. 4(a)).

Dose-response analysis. Six studies from five publications were included in the dose-response analysis of whole-grain consumption and total cancer mortality risk (Fig. 4(b)). The SRR per 3 servings/d was 0.91 (95\% CI 0.84, 0.98), with evidence of high heterogeneity $\left(I^{2}=78.4 \%, P_{\text {heterogeneity }}<0 \cdot 001\right)$.

\section{Subgroup, meta-regression and sensitivity analyses}

Subgroup and meta-regression analyses are shown in Table 2. Overall, only study location modified the association between whole-grain intake and cancer mortality (USA: SRR 0.95; $95 \% \mathrm{CI}$ $0.91,0.99$; Europe: SRR $0.78 ; 95 \%$ CI $0.70,0.87 ; P_{\text {for difference }}=$ 0.011). However, other variables, such as sex, duration of followup, baseline mean age and adjustments for DM, dyslipidaemia and hypertension did not significantly alter these associations. For example, there were significant inverse associations for both women (SRR 0.85; 95\% CI 0.81, 0.90; $P_{\text {heterogeneity }}=0.052 ; I^{2}=61.1 \%$ ) and men (SRR 0.89; 95\% CI 0.83, 0.96; $P_{\text {heterogeneity }}=0.018$; $\left.I^{2}=75.0 \%\right)$ for the association between whole grains and all-cause mortality. Stratified analyses by duration of follow-up showed a similar risk relationship. Meta-regression analysis showed that no variables may account for the heterogeneity across studies on the association between whole-grain intake and total mortality. 
Table 1. Characteristics of the studies of the association between whole-grain consumption and risk of all-cause and cause-specific mortality (Risk estimations and $95 \%$ confidence intervals (highest $v$. lowest))

\begin{tabular}{|c|c|c|c|c|c|c|c|c|}
\hline $\begin{array}{l}\text { First author/ } \\
\text { publication year/ } \\
\text { country }\end{array}$ & Study characteristics & $\begin{array}{l}\text { Dietary } \\
\text { assessment }\end{array}$ & Outcome $(n)$ & Exposure details & Risk estimation & $95 \% \mathrm{Cl}$ & Adjustments & NOS \\
\hline \multirow{5}{*}{$\begin{array}{l}\text { Jacobs/2001/ } \\
\text { Norway }\end{array}$} & \multirow{5}{*}{$\begin{array}{l}\text { NCS } \\
n 33848, \mathrm{M}+\mathrm{F} \\
\text { Mean age: } 46 \text { years } \\
\text { Follow-up, } 14.4 \text { years }\end{array}$} & \multirow{5}{*}{$\begin{array}{l}\text { Whole-grain } \\
\text { bread score } \\
\text { FFQ-66 items }\end{array}$} & \multirow{5}{*}{$\begin{array}{l}\text { Total mortality } \\
\quad(1991)\end{array}$} & $0.05-0.60$ & \multicolumn{2}{|c|}{1.00} & \multirow{5}{*}{$\begin{array}{l}\text { Age, energy intake, sex smoking, physical } \\
\text { activity, use of cod liver oil, multivitamins, SFA } \\
\text { intake, systolic blood pressure, serum total } \\
\text { cholesterol, BMl }\end{array}$} & \multirow[t]{5}{*}{8} \\
\hline & & & & $0.83-0.83$ & 0.87 & $0.75,1.01$ & & \\
\hline & & & & $0.90-1.13$ & 0.80 & $0.71,0.92$ & & \\
\hline & & & & $1.35-1.80$ & 0.85 & $0.74,0.98$ & & \\
\hline & & & & $2 \cdot 25-5 \cdot 40$ & 0.75 & $0.65,0.88$ & & \\
\hline \multirow[t]{4}{*}{ Liu/2003/USA } & \multirow{4}{*}{$\begin{array}{l}\text { PHS } \\
n 86190, \mathrm{M} \\
\text { Mean age: } 56 \text { years } \\
\text { Follow-up, } 5.5 \text { years }\end{array}$} & \multirow{4}{*}{$\begin{array}{l}\text { Validated } \\
\text { FFQ-126 items }\end{array}$} & \multirow{4}{*}{$\begin{array}{l}\text { Total mortality } \\
\quad(3114)\end{array}$} & Rarely & \multicolumn{2}{|c|}{1.00} & \multirow{4}{*}{$\begin{array}{l}\text { Age; cigarette smoking; alcohol intake; physical } \\
\text { activity; BMI; type } 2 \text { diabetes, high cholesterol, } \\
\text { hypertension; and use of multivitamins }\end{array}$} & \multirow[t]{4}{*}{8} \\
\hline & & & & 1 servings/week & 0.88 & $0.76,1.01$ & & \\
\hline & & & & $\begin{array}{l}\text { 2-6 servings/ } \\
\text { week }\end{array}$ & 0.85 & $0.74,0.97$ & & \\
\hline & & & & $\geq 1$ serving/d & 0.83 & $0.73,0.94$ & & \\
\hline \multirow[t]{5}{*}{ Steffen/2003/USA } & \multirow{5}{*}{$\begin{array}{l}\text { ARIC } \\
n 11940, \mathrm{M}+\mathrm{F} \\
\text { Mean age: } 53 \text { years } \\
\text { Follow-up, } 11 \text { years }\end{array}$} & \multirow{5}{*}{$\begin{array}{l}\text { Validated FFQ-61 } \\
\text { items }\end{array}$} & \multirow{5}{*}{$\begin{array}{l}\text { Total mortality } \\
\quad(867)\end{array}$} & 0.1 serving/d & \multicolumn{2}{|c|}{1.00} & \multirow{5}{*}{$\begin{array}{l}\text { Age, race, sex, energy intake education, smoking } \\
\text { status, physical activity, alcohol intake and } \\
\text { hormone replacement, BMI, waist:hip ratio, } \\
\text { systolic blood pressure, use of } \\
\text { antihypertensive medications }\end{array}$} & \multirow[t]{5}{*}{9} \\
\hline & & & & 0.5 serving $/ d$ & 0.96 & $0.79,1.17$ & & \\
\hline & & & & 1 serving $/ \mathrm{d}$ & 0.80 & $0.65,0.99$ & & \\
\hline & & & & 1.5 serving/d & 0.87 & $0.70,1.08$ & & \\
\hline & & & & 3.0 serving/d & $0 \cdot 77$ & $0.61,0.97$ & & \\
\hline \multirow{4}{*}{$\begin{array}{l}\text { Sahyoun/2006/ } \\
\text { USA }\end{array}$} & \multirow{4}{*}{$\begin{array}{l}\text { Community-living } \\
\text { persons } \\
n 535, \mathrm{M}+\mathrm{F} \\
\text { Mean age: } 72 \text { years } \\
\text { Follow-up, } 12-15 \text { years }\end{array}$} & \multirow[t]{4}{*}{ 3-d food record } & \multirow{4}{*}{$\begin{array}{l}\text { Total mortality } \\
\text { (185) }\end{array}$} & 0.31 serving/d & \multicolumn{2}{|c|}{1.00} & \multirow{4}{*}{$\begin{array}{l}\text { Age, sex, ethnicity, educational attainment, } \\
\text { marital status, smoking, alcohol intake, } \\
\text { exercise, BMI, energy intake, percentage SFA } \\
\text { intake, history of heart disease and use of } \\
\text { antihypertensive or lipid-lowering medication }\end{array}$} & \multirow[t]{4}{*}{8} \\
\hline & & & & 0.86 serving/d & 1.08 & $0.71,1.66$ & & \\
\hline & & & & 1.49 serving/d & 1.24 & $0.83,1.86$ & & \\
\hline & & & & 2.9 serving/d & 0.82 & $0.52,1 \cdot 28$ & & \\
\hline \multirow[t]{5}{*}{ Jacobs/2007/USA } & $\begin{array}{l}\text { IWHS } \\
n 27,312, \mathrm{~F}\end{array}$ & $\begin{array}{l}\text { Validated } \\
\text { FFQ-127 items }\end{array}$ & $\begin{array}{l}\text { Total mortality } \\
\text { (5552) }\end{array}$ & $\begin{array}{l}<3.5 \text { servings/ } \\
\text { week }\end{array}$ & & & $\begin{array}{l}\text { Age, energy intake, BMI, waist:hip ratio, smoking, } \\
\text { education, physical activity, oestrogen use, }\end{array}$ & 8 \\
\hline & $\begin{array}{l}\text { Mean age: } 61 \text { years } \\
\text { Follow-up, } 17 \text { years }\end{array}$ & & & $\begin{array}{l}\text { 4-7 servings/ } \\
\text { week }\end{array}$ & 0.88 & $0.81,0.96$ & & \\
\hline & & & & $\begin{array}{l}7.5-10 \cdot 5 \\
\quad \text { servings/week }\end{array}$ & 0.88 & $0.81,0.96$ & and seafood, total fruit and vegetables & \\
\hline & & & & $\begin{array}{l}11-18.5 \text { servings/ } \\
\text { week }\end{array}$ & $0 \cdot 80$ & $0.73,0.87$ & & \\
\hline & & & & $\begin{array}{l}>19 \text { servings/ } \\
\text { week }\end{array}$ & 0.79 & $0.72,0.87$ & & \\
\hline Buil-Cosiales/ & PREDIMED & Validated & Total mortality & $0 \mathrm{~g} / \mathrm{d}$ & & & Age, sex, smoking, diabetes, BMI, blood & 8 \\
\hline 2014/Spain & $\begin{array}{l}n 7216, \mathrm{M}+\mathrm{F} \\
\text { Mean age: } 67 \text { vears }\end{array}$ & FFQ-137 & (425) & $5 \mathrm{~g} / \mathrm{d}$ & 0.78 & $0.52,1.17$ & $\begin{array}{l}\text { pressures, use of statins, alcohol intake, } \\
\text { educational level, physical activity, total energy }\end{array}$ & \\
\hline & Follow up, 5.9 years & & & $\begin{array}{l}19 \mathrm{~g} / \mathrm{d} \\
84 \mathrm{~g} / \mathrm{d}\end{array}$ & $\begin{array}{l}0.67 \\
0.92\end{array}$ & $\begin{array}{l}0.43,1.04 \\
0.64,1.33\end{array}$ & intake, fruit and vegetable consumption & \\
\hline Huang/2015/USA & NIH-AARP Diet and & Self-administered & Total mortality & $0.13 \mathrm{oz} e q / \mathrm{d}$ & & & Age, sex, smoking, race, alcohol intake, & 9 \\
\hline & Health Study & FFQ-124 & $(46067)$ & $0.30 \mathrm{oz} e q / \mathrm{d}$ & 0.96 & $0.93,0.99$ & education level, marital status, health status, & \\
\hline & $n 367442, \mathrm{M}+\mathrm{F}$ & & & $0.47 \mathrm{oz} e \mathrm{eq}$ & 0.95 & $0.92,0.98$ & obesity, physical activity, consumption of red & \\
\hline & Mean age: 61 years & & & $0.69 \mathrm{oz} e q / \mathrm{d}$ & 0.92 & $0.89,0.96$ & meat, total fruit and total vegetables, total & \\
\hline & Follow up, 14 years & & & $1.20 \mathrm{oz} e \mathrm{e} / \mathrm{d}$ & 0.94 & $0.90,0.97$ & energy intake and hormone usage, cereal fibre & \\
\hline
\end{tabular}




\section{N British Journal of Nutrition}

\begin{tabular}{|c|c|c|c|c|c|c|c|c|}
\hline $\begin{array}{l}\text { First author/ } \\
\text { publication year/ } \\
\text { country }\end{array}$ & Study characteristics & $\begin{array}{l}\text { Dietary } \\
\text { assessment }\end{array}$ & Outcome $(n)$ & Exposure details & Risk estimation & $95 \% \mathrm{Cl}$ & Adjustments & NOS \\
\hline Wu/2015/USA & $\begin{array}{l}\text { NHS } \\
n 74341, \mathrm{~F} \\
\text { Mean age: } 50 \text { years } \\
\text { Follow-up, } 26 \text { years }\end{array}$ & $\begin{array}{l}\text { Validated } \\
\text { FFQ-126 items }\end{array}$ & $\begin{array}{l}\text { Total mortality } \\
\quad(15106)\end{array}$ & $\begin{array}{l}4.2 \mathrm{~g} / \mathrm{d} \\
9.7 \mathrm{~g} / \mathrm{d} \\
14.7 \mathrm{~g} / \mathrm{d} \\
21.1 \mathrm{~g} / \mathrm{d} \\
33 \mathrm{~g} / \mathrm{d}\end{array}$ & $\begin{array}{l}0.98 \\
1.00 \\
0.94 \\
0.88\end{array}$ & $\begin{array}{l}0.93,1.03 \\
0.95,1.05 \\
0.89,0.99 \\
0.84,0.93\end{array}$ & $\begin{array}{l}\text { Age; cigarette smoking; alcohol intake; physical } \\
\text { activity; BMI; history of type } 2 \text { diabetes, high } \\
\text { cholesterol and hypertension; and use of } \\
\text { multivitamins; healthy eating index }\end{array}$ & 8 \\
\hline Wu/2015/USA & $\begin{array}{l}\text { HPFS } \\
n 43,744, \mathrm{M} \\
\text { Mean age: } 53 \text { years } \\
\text { Follow-up, } 24 \text { years }\end{array}$ & $\begin{array}{l}\text { Validated } \\
\quad \text { FFQ-126 items }\end{array}$ & $\begin{array}{l}\text { Total mortality } \\
\quad(11814)\end{array}$ & $\begin{array}{l}5.9 \mathrm{~g} / \mathrm{d} \\
14.4 \mathrm{~g} / \mathrm{d} \\
22.1 \mathrm{~g} / \mathrm{d} \\
31.3 \mathrm{~g} / \mathrm{d} \\
47.8 \mathrm{~g} / \mathrm{d}\end{array}$ & $\begin{array}{l}1.00 \\
0.97 \\
1.01 \\
0.95\end{array}$ & $\begin{array}{l}0.94,1.05 \\
0.91,1.02 \\
0.95,1.07 \\
0.89,1.00\end{array}$ & $\begin{array}{l}\text { Age; cigarette smoking; alcohol intake; physical } \\
\text { activity; BMI; history of eating index }\end{array}$ & 8 \\
\hline Boggs/2015/USA & $\begin{array}{l}\text { BWHS } \\
n 37001, \mathrm{~F} \\
\quad \text { Mean age: } 42 \text { years } \\
\text { Follow-up, } 16 \text { years }\end{array}$ & Validated FFQ & $\begin{array}{l}\text { Total mortality } \\
\quad(1678)\end{array}$ & $\begin{array}{l}0.01 \text { serving/d } \\
0.11 \text { serving/d } \\
0.32 \text { serving/d } \\
0.62 \text { serving/d } \\
1.44 \text { serving/d }\end{array}$ & $\begin{array}{l}0.96 \\
0.82 \\
0.85 \\
0.75\end{array}$ & $\begin{array}{l}0.83,1.12 \\
0.70,0.97 \\
0.73,0.99 \\
0.64,0.89\end{array}$ & $\begin{array}{l}\text { Age, each DASH component, total energy intake, } \\
\text { education, marital status, vigorous exercise, } \\
\text { television watching, smoking and alcohol } \\
\text { intake }\end{array}$ & 8 \\
\hline $\begin{array}{l}\text { Johnsen/2015/ } \\
\text { Scandinavian }\end{array}$ & $\begin{array}{l}\text { HELGA cohort } \\
n 120009 \\
\text { Mean age: } 51 \text { years }(\mathrm{F}) \\
\quad 54 \text { years }(\mathrm{M}) \\
\text { Follow-up, } 14.2 \text { years }\end{array}$ & $\begin{array}{l}\text { Validated } \\
\text { FFQ-173 }\end{array}$ & $\begin{array}{l}\text { Total mortality } \\
\quad(7839)\end{array}$ & $\begin{array}{l}56 \mathrm{~g} / \mathrm{d} \\
100 \mathrm{~g} / \mathrm{d} \\
131 \mathrm{~g} / \mathrm{d} \\
201 \mathrm{~g} / \mathrm{d} \\
64 \mathrm{~g} / \mathrm{d} \\
107 \mathrm{~g} / \mathrm{d} \\
156 \mathrm{~g} / \mathrm{d} \\
222 \mathrm{~g} / \mathrm{d}\end{array}$ & $\begin{array}{l}0.78 \\
0.77 \\
0.68 \\
\\
\\
0.87 \\
0.74 \\
0.75\end{array}$ & $\begin{array}{l}\mathrm{F} \\
0.71,0.86, \mathrm{~F} \\
0.71,0.85, \mathrm{~F} \\
0.62,0.75, \mathrm{~F} \\
\mathrm{M} \\
0.80,0.95, \mathrm{M} \\
0.68,0.81, \mathrm{M} \\
0.68,0.81, \mathrm{M}\end{array}$ & $\begin{array}{l}\text { Age, follow-up time, education, smoking, alcohol, } \\
\text { BMI, total energy intake }\end{array}$ & 9 \\
\hline
\end{tabular}

NOS, Newcastle-Ottawa Scale; NCS, Norwegian County Study; M, male; F, female; PHS, Physicians' Health Study; ARIC, Atherosclerosis Risk in Communities Study; IWHS, lowa Women's Health Study; PREDIMED, Prevención con Dieta Mediterránea; NIH-AARP, National Institutes of Health-American Association of Retired Persons; NHS, Nurses' Health Study; HPFS, Health Professionals Follow-Up Study; BWHS, Black Women's Health Study; DASH, Dietary Approaches to Stop Hypertension. 
(a)

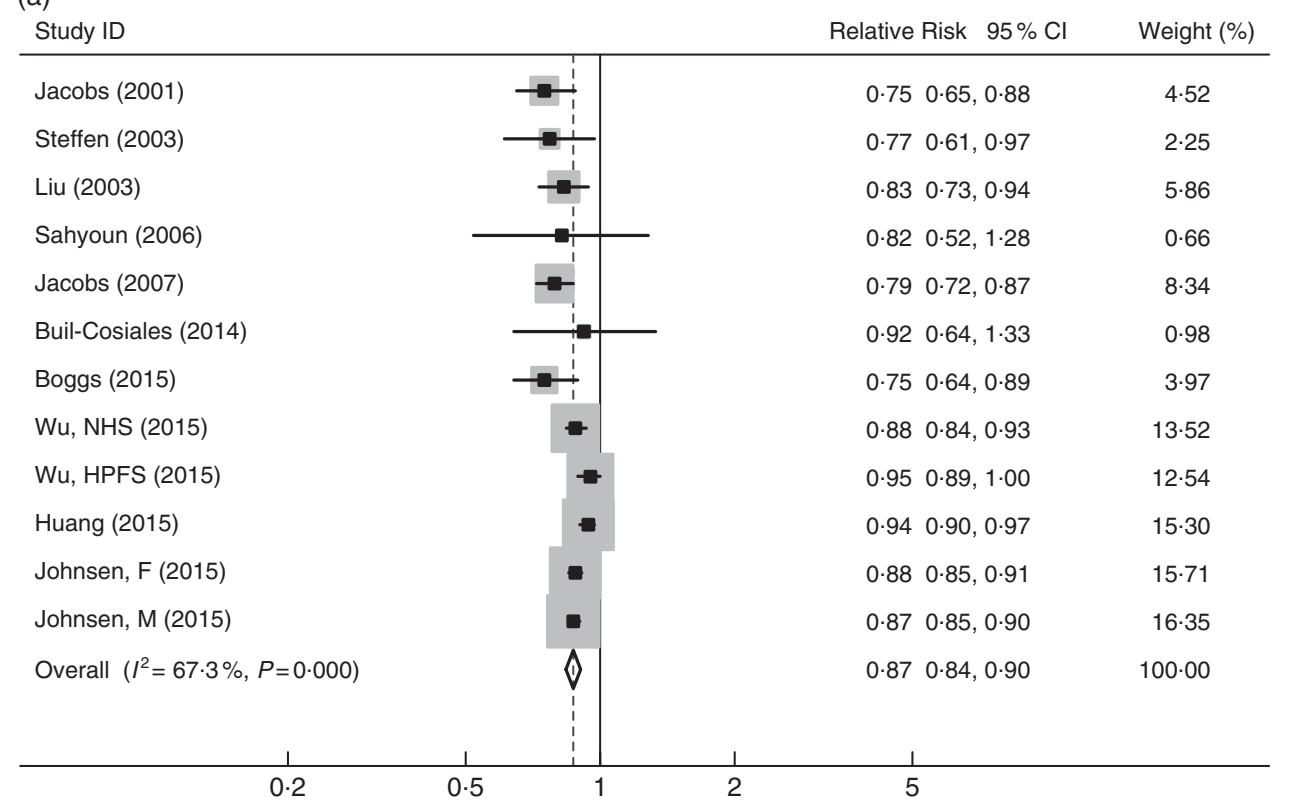

(b)

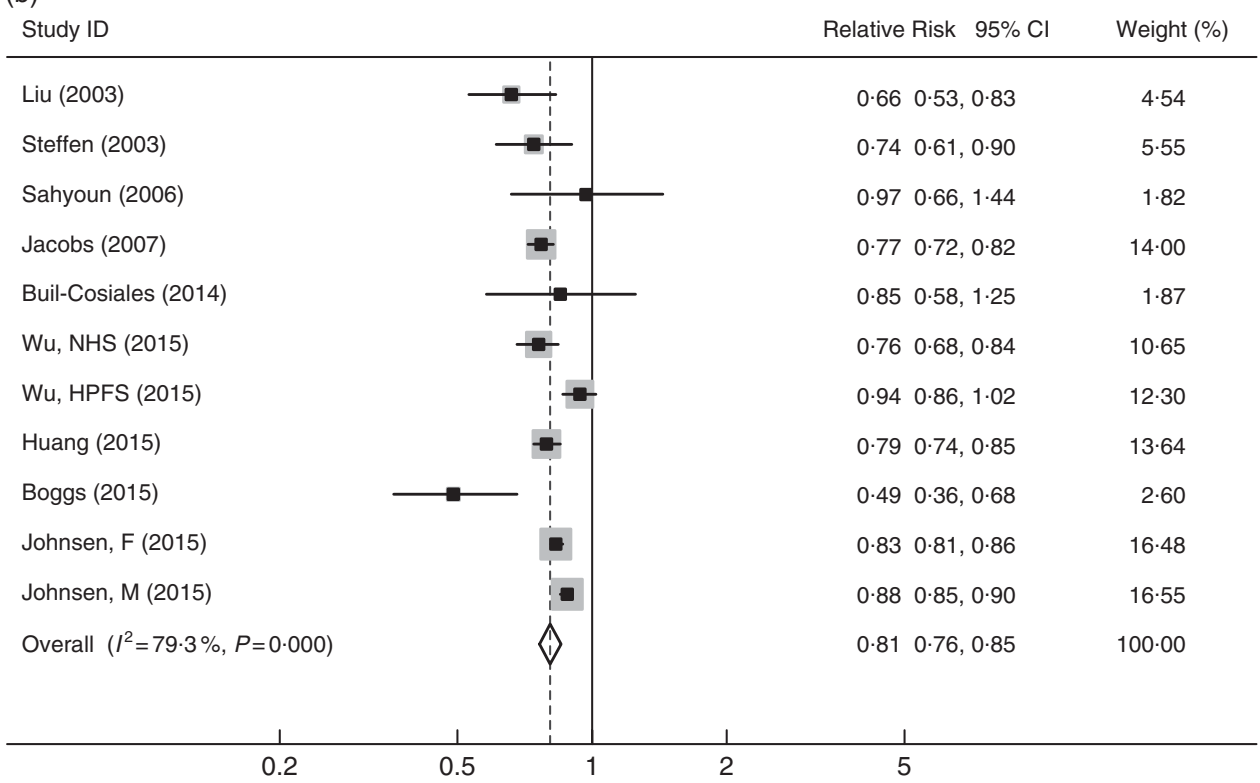

Fig. 2. The summary risk association between the intake of whole grains and all-cause mortality according to (a) the highest $v$. lowest analysis; (b) linear dose-response analysis (per 3 serving/d). Weights are from random-effects analysis. NHS, Nurses' Health Study; HPFS, Health Professionals Follow-Up Study; $\mathrm{F}$, female; $\mathrm{M}$, male.

We also conducted a sensitivity analysis by omitting one study at a time and calculating the pooled RR for the remainder of studies, and we found that there were no changes in the direction of effect when any one study was excluded (data not shown). For example, by excluding one study that reported findings for the consumption of whole-grain breakfast cereals ${ }^{(11)}$, there were significant associations between whole-grain intake and all-cause mortality (SRR 0.87; $95 \% \quad$ CI $\left.\quad 0.84, \quad 0.91 ; \quad P_{\text {heterogeneity }}=0.001 ; \quad I^{2}=69.4 \%\right)$. On excluding one study that included participants with the evidently older baseline age (mean: 72 years) ${ }^{(17)}$, the significant associations did not change. Moreover, repeated analysis of high $v$. low intake using the studies included in the linear dose-response analysis for intake produced results that were similar to those of the original analysis (data not shown).

\section{Non-linear dose-response analyses}

There was evidence of a non-linear association between whole-grain intake and the risk of mortality from all causes $\left(P_{\text {non-linearity }}<0 \cdot 001\right.$, online Supplementary Fig. S1A $)$ and CVD 
(a)

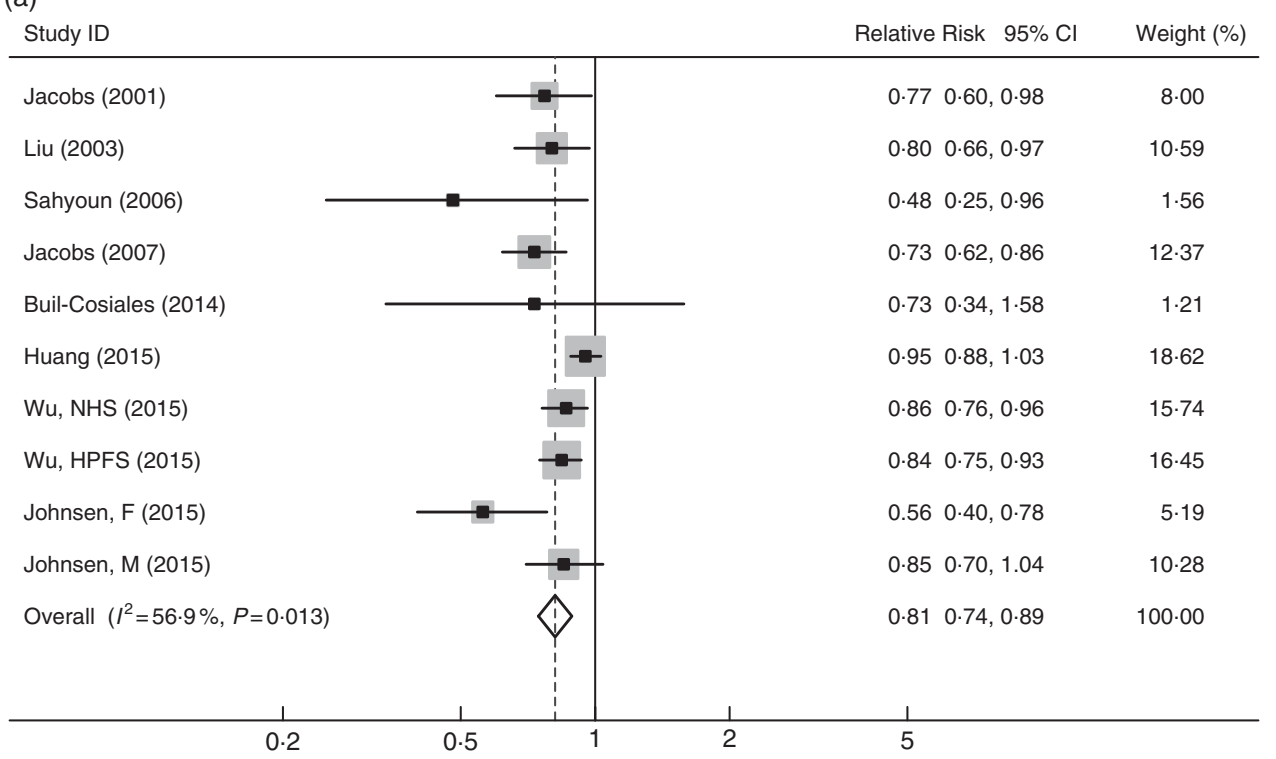

(b)

\begin{tabular}{|c|c|c|c|c|}
\hline Study ID & & & Relative Risk $95 \% \mathrm{Cl}$ & Weight (\%) \\
\hline Liu (2003) & & & $0.610 .43,0.85$ & 6.90 \\
\hline Sahyoun (2006) & 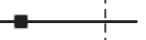 & & $0.48 \quad 0.27,0.87$ & 3.08 \\
\hline Jacobs (2007) & -- & & $0.750 .67,0.84$ & $15 \cdot 89$ \\
\hline Buil-Cosiales (2014) & & & $0.680 .31,1.53$ & 1.79 \\
\hline Huang (2015) & & & $0.780 .67,0.90$ & $14 \cdot 27$ \\
\hline Wu, NHS (2015) & -7 & & $0.610 .48,0.78$ & $10 \cdot 05$ \\
\hline Wu, HPFS (2015) & & & $0.72 \quad 0.62,0.84$ & $14 \cdot 07$ \\
\hline Johnsen, F (2015) & & & $0.760 .68,0.85$ & 15.96 \\
\hline Johnsen, M (2015) & & & $0.92 \quad 0.87,0.98$ & 17.99 \\
\hline Overall $\left(I^{2}=76.3 \%, P=0.000\right)$ & & & $0.740 .66,0.83$ & $100 \cdot 00$ \\
\hline 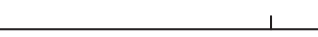 & 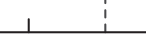 & & $\perp$ & \\
\hline
\end{tabular}

Fig. 3. The summary risk association between the intake of whole grains and CVD mortality according to (a) the highest $v$. lowest analysis; (b) linear dose-response analysis (per 3 serving/d). Weights are from random-effects analysis. NHS, Nurses' Health Study; HPFS, Health Professionals Follow-Up Study; F, female; M, male.

$\left(P_{\text {non-linearity }}=0 \cdot 003\right.$, online Supplementary Fig. S1B $)$. However, there was no evidence of a non-linear association between whole-grain intake and the risk of mortality from cancer $\left(P_{\text {non-linearity }}=0 \cdot 170\right.$, online Supplementary Fig. S1C).

\section{Publication biases}

Egger's test revealed the publication bias $(P=0.002)$ for the association between whole-grain consumption and all-cause mortality risk, whereas Begg's test did not confirm this $(P=0.945$; online Supplementary Fig. S2A). The trim-and-fill method suggested that no additional risk estimate was required to balance the funnel plot. In case of the association with CVD death, there was no indication of publication bias by both Egger's test $(P=0.834)$ and Begg's test $(P=0 \cdot 107$; online Supplementary Fig. S2B). For the association with cancer death, there was no indication of publication bias by both Egger's test $(P=0.959)$ and Begg's test $(P=0 \cdot 174$; online Supplementary Fig. S2C).

\section{Discussion}

In this meta-analysis, we found that higher consumption of whole grains was associated with a decreased risk of all-cause, CVD and cancer mortality; eating an additional three servings of whole grains daily reduced all-cause mortality by $19 \%$, CVD 
(a)

\begin{tabular}{|c|c|c|c|}
\hline Study ID & & Relative Risk $95 \% \mathrm{Cl}$ & Weight (\%) \\
\hline Jacobs (2001) & . & $0.790 .62,1.02$ & $6 \cdot 51$ \\
\hline Jacobs (2007) & & $0.89 \quad 0.77,1.04$ & 12.05 \\
\hline Buil-Cosiales (2014) & & $0.75 \quad 0.40,1.41$ & 1.32 \\
\hline Johnsen, F (2015) & $\rightarrow$ & $0.860 .74,0.99$ & $12 \cdot 43$ \\
\hline Johnsen, M (2015) & $\rightarrow$ & $0.70 \quad 0.60,0.81$ & $12 \cdot 07$ \\
\hline Huang (2015) & $=$ & $0.930 .88,0.99$ & 20.57 \\
\hline Wu, NHS (2015) & - & $0.990 .91,1.07$ & $18 \cdot 46$ \\
\hline Wu, HPFS (2015) & $\rightarrow-$ & $0.950 .86,1.05$ & $16 \cdot 59$ \\
\hline Overall $\left(I^{2}=63.6 \%, P=0.007\right)$ & \langle & $0.890 .82,0.96$ & $100 \cdot 00$ \\
\hline 1 & 1 & $\perp$ & \\
\hline
\end{tabular}

(b)

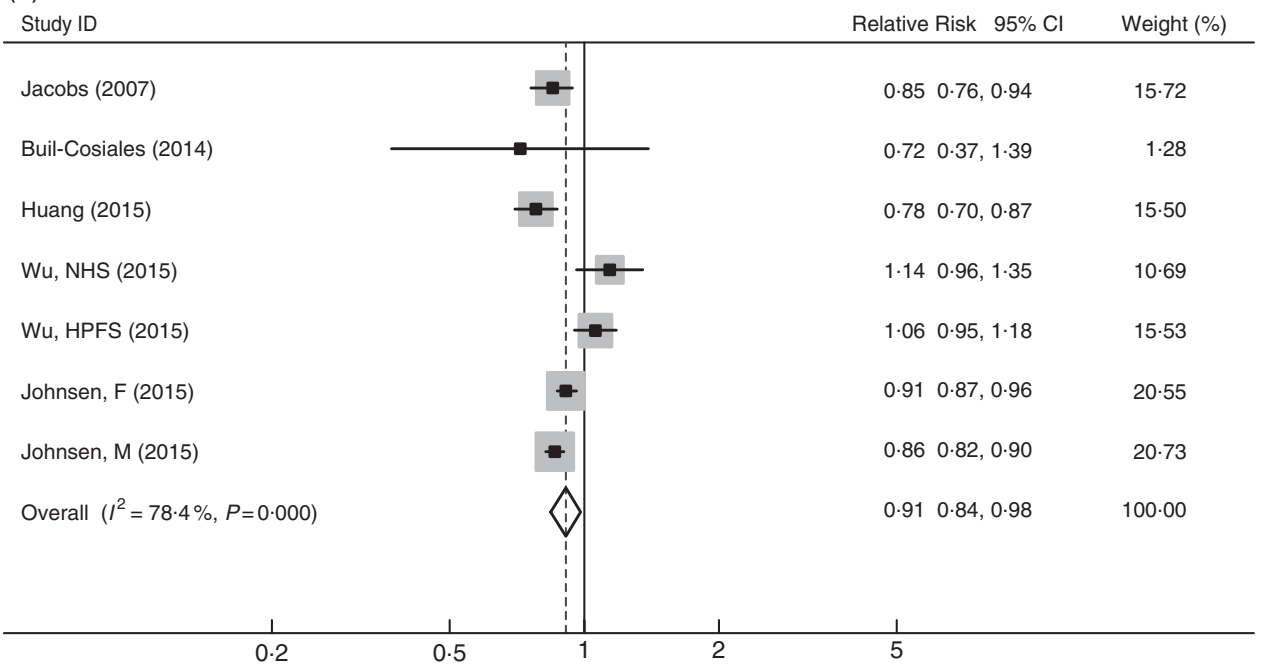

Fig. 4. The summary risk association between the intake of whole grains and cancer mortality according to (a) the highest $v$. lowest analysis; (b) linear dose-response analysis (per 3 serving/d). Weights are from random-effects analysis. F, Female; M, male; NHS, Nurses' Health Study; HPFS, Health Professionals Follow-Up Study.

death by $26 \%$ and cancer death by $9 \%$. There was evidence of a non-linear risk association between whole-grain intake and the risk of mortality due to all causes and CVD, but a linear risk association with the risk of mortality due to cancer.

Clinical trials have suggested that consumption of whole grains may positively affect risk factors for CVD and cancer, including hypertension ${ }^{(31,32)}$, weight gain/obesity ${ }^{(4,5,33,34)}$, total and LDL-cholesterol ${ }^{(35,36)}$ and type $2 \mathrm{DM}^{(6,7,37,38)}$. For instance, Magnusdottir et al. ${ }^{(35)}$ found that increased consumption of whole-grain rye was associated with lower blood levels of LDL-cholesterol. A randomised cross-over trial found that, when compared with refined wheat crisp bread, consumption of whole-grain rye crisp bread is associated with lower postprandial glucose and insulin responses ${ }^{(38)}$.

Whole-grain intake may lower all-cause mortality rates by decreasing the risk of major chronic diseases, including
$\operatorname{CVD}^{(8,36)}$ and some cancers ${ }^{(8,39,40)}$, which are significant causes of mortality worldwide. On the basis of ten prospective studies, Ye et $a l .{ }^{(36)}$ found that, compared with never/rare consumers of whole grains, those consuming 3-5 servings of whole grain/d had a $21 \%$ lower risk of CVD (RR 0.79; $95 \%$ CI $0.74,0.85$ ). Associations between whole-grain intakes and cancer mortality remain inconclusive. Several previous reports ${ }^{(13,14,18)}$ have found that consumption of whole grains was inversely associated with the risk of cancer death. These associations were attenuated after further adjustment for dietary factors and other healthy lifestyle factors. With regard to the association with various cancer deaths, some researchers observed an inverse association between whole-grain consumption and risk of mortality from colorectal cancer in men. However, they did not find significant associations with mortality from prostate cancer, breast cancer or lung cancer ${ }^{(14)}$. In addition, the associations 
Table 2. Subgroup and meta-regression analyses for the association between whole-grain consumption and all-cause, CVD and cancer mortality (Relative risks (RR) and 95\% confidence intervals)

\begin{tabular}{|c|c|c|c|c|c|c|c|c|c|c|c|c|c|c|c|}
\hline \multirow[b]{2}{*}{ Subgroup } & \multicolumn{5}{|c|}{ All-cause } & \multicolumn{5}{|c|}{ CVD } & \multicolumn{5}{|c|}{ Total cancer } \\
\hline & No. & $\mathrm{RR}$ & $95 \% \mathrm{Cl}$ & $P_{\mathrm{h}} ; P(\%)$ & $P_{\mathrm{d}}$ & No. & $\mathrm{RR}$ & $95 \% \mathrm{Cl}$ & $P_{\mathrm{h}} ; P(\%)$ & $P_{\mathrm{d}}$ & No. & $\mathrm{RR}$ & $95 \% \mathrm{Cl}$ & $P_{\mathrm{h}} ; P(\%)$ & $P_{\mathrm{d}}$ \\
\hline All & 11 & 0.87 & $0.84,0.90$ & $<0.001 ; 67.3$ & & 9 & 0.81 & $0.75,0.89$ & $0.013 ; 56.9$ & & 7 & 0.89 & $0.82,0.96$ & $0.007 ; 63.6$ & \\
\hline Sex & & & & & 0.362 & & & & & 0.231 & & & & & 0.351 \\
\hline Men & 3 & 0.89 & $0.83,0.96$ & $0.018 ; 75.0$ & & 3 & 0.83 & $0.77,0.91$ & $0.890 ; 0$ & & 2 & 0.82 & $0.61,1 \cdot 11$ & $0.001 ; 90.9$ & \\
\hline Women & 4 & 0.85 & $0.81,0.90$ & $0.052 ; 61.1$ & & 3 & 0.74 & $0.61,0.90$ & $0.03 ; 71.4$ & & 3 & 0.93 & $0.85,1.02$ & $0.175 ; 42.7$ & \\
\hline Location & & & & & 0.804 & & & & & 0.325 & & & & & 0.211 \\
\hline USA & 8 & 0.87 & $0.82,0.92$ & $0.001 ; 71.5$ & & 6 & 0.84 & $0.76,0.92$ & $0.02 ; 62.7$ & & 4 & 0.85 & $0.71,0.99$ & $0.537 ; 0$ & \\
\hline Europe & 3 & 0.87 & $0.84,0.90$ & $0.242 ; 28.3$ & & 3 & 0.75 & $0.62,0.90$ & $0.216 ; 32.7$ & & 3 & 0.78 & $0.70,0.87$ & $0.290 ; 0$ & \\
\hline Baseline mean age (years) & & & & & 0.831 & & & & & 0.712 & & & & & 0.379 \\
\hline$\leq 53$ & 5 & 0.86 & $0.82,0.92$ & $0.008 ; 68.1$ & & 4 & 0.80 & $0.71,0.90$ & $0.108 ; 50.6$ & & 3 & 0.93 & $0.86,1.01$ & $0.181 ; 38.5$ & \\
\hline$>53$ & 6 & 0.87 & $0.82,0.92$ & $0.003 ; 72 \cdot 2$ & & 5 & 0.82 & $0.71,0.94$ & $0.023 ; 61.7$ & & 4 & 0.84 & $0.72,0.98$ & $0.007 ; 75.5$ & \\
\hline Follow-up (years) & & & & & 0.915 & & & & & 0.869 & & & & & 0.712 \\
\hline$\leq 14$ & 5 & 0.86 & $0.77,0.96$ & $0.008 ; 70 \cdot 8$ & & 5 & 0.80 & $0.67,0.96$ & $0.012 ; 69.0$ & & 3 & 0.92 & $0.87,0.98$ & $0.703 ; 0$ & \\
\hline$>14$ & 6 & 0.87 & $0.84,0.91$ & $0.012 ; 63.3$ & & 4 & 0.82 & $0.74,0.90$ & $0.188 ; 34.9$ & & 4 & 0.87 & $0.76,0.98$ & $0.001 ; 78.3$ & \\
\hline \multicolumn{16}{|l|}{ Adjustments } \\
\hline Diabetes, yes & 5 & 0.87 & $0.81,0.94$ & $0.015 ; 71.9$ & 0.711 & 5 & 0.82 & $0.77,0.88$ & $0.571 ; 0$ & 0.998 & 4 & 0.96 & $0.91,1.02$ & $0.540 ; 0$ & 0.178 \\
\hline No & 6 & 0.87 & $0.83,0.91$ & $0.015 ; 67.4$ & & 4 & 0.77 & $0.63,0.94$ & $0.005 ; 72.9$ & & 3 & 0.83 & $0.72,0.95$ & $0.005 ; 77.0$ & \\
\hline Hypertension, yes & 7 & 0.87 & $0.81,0.93$ & $0.047 ; 52.9$ & 0.991 & 6 & 0.83 & $0.77,0.89$ & $0.615 ; 0$ & 0.987 & 4 & 0.95 & $0.89,1.03$ & $0.315 ; 15.3$ & 0.334 \\
\hline No & 4 & 0.87 & $0.83,0.91$ & $<0.001 ; 80.8$ & & 3 & 0.79 & $0.65,0.96$ & $0.001 ; 81.0$ & & 3 & 0.85 & $0.75,0.96$ & $0.007 ; 75.4$ & \\
\hline Dyslipidaemia, yes & 4 & 0.87 & $0.80,0.94$ & $0.012 ; 72.8$ & 0.799 & 3 & 0.84 & $0.78,0.90$ & $0.834 ; 0$ & 0.673 & 3 & 0.95 & $88,1.03$ & $0.229 ; 32.2$ & 0.278 \\
\hline No & 7 & 0.87 & $0.83,0.91$ & $0.002 ; 68.7$ & & 6 & 0.76 & $0.63,0.92$ & $0.002 ; 73.9$ & & 4 & 0.85 & $0.75,0.95$ & $0.014 ; 67.9$ & \\
\hline
\end{tabular}

$P_{\mathrm{h}}, P_{\text {heterogeneity }} ; P_{\mathrm{d}}, P_{\text {for difference. }}$ 
with cancer incidence may depend on the population characteristics and vary by specific types of cancer. An analysis of six prospective cohort studies found that the RR of colorectal cancer was 0.83 (95\% CI $0.78,0.89)$ per increment of three servings of whole grains daily ${ }^{(40)}$. However, no significant associations were found for incidence of ovarian cancer, prostate cancer, breast cancer or endometrial cancer ${ }^{(41-43)}$.

Several mechanisms may explain the inverse association between whole-grain consumption and all-cause mortality. Both the bran and germ layers of whole grains are high in fibres, antioxidants, phytochemicals and minerals (including $\mathrm{Mg}, \mathrm{K}$ and $\mathrm{Ca}$ ). Dietary fibre consumption is inversely associated with the risk of a number of chronic diseases, including $\mathrm{CHD}^{(44)}$, stroke, colorectal adenoma and carcinoma $^{(27,45)}$ and breast cancer ${ }^{(46)}$. Dietary fibre has been shown to increase the production of SCFA and the rate of bile excretion, leading to reduced serum cholesterol levels ${ }^{(47)}$. In addition, it is postulated that dietary fibre may reduce the risk of bowel cancer by reducing the contact time between potential carcinogens and mucosal cells, and by increasing the binding of carcinogens to bile acids $^{(48)}$. Observational studies and clinical trials have shown that an increased intake of $\mathrm{Mg}$ and $\mathrm{K}$ is inversely associated with the risk of certain chronic diseases ${ }^{(49,50)}$. Whole grains are rich in these nutrients.

To the best of our knowledge, this is the first meta-analysis to explore the relationship between whole-grain consumption and the risk of all-cause mortality. All of the studies included in this meta-analysis were prospective cohort studies, which minimises recall and selection biases. The meta-analysis included more than 810000 subjects and nearly 90000 cases of all-cause mortality. The large numbers meant that it had sufficient statistical power to detect moderate associations. All of the studies included in this meta-analysis were of high quality. Moreover, the majority adjusted for important confounders, such as smoking, alcohol, physical activity, BMI and intermediate biomarkers, such as DM, hypertension and dyslipidaemia. Finally, we were able to confirm our findings using both linear and non-linear dose-response analyses.

However, our study had some limitations. Measurement errors were inevitable, as it is very difficult for individuals to accurately report their intake of whole grains. All the included studies used total whole grains as a category, and we excluded studies that investigated only specific types of whole grains, such as brown rice ${ }^{(51)}$. The aggregation of consumption data may have masked the beneficial effects of specific whole grains. In addition, the definition of whole grains differed somewhat between studies. For example, one considered breakfast cereals to be made of whole grains if the product contained $>25 \%$ whole grain or bran by weight ${ }^{(11)}$, whereas some studies did not provide definitions of whole grains. On the other hand, whole-grain intake was determined using self-administered FFQ in all but one study. This may have resulted in inaccuracies, although it is reassured in one cohort study ${ }^{(52)}$, which found that when compared with a 7-d dietary record assessment of whole-grain consumption by the use of FFQ had relatively high validity $(r>0 \cdot 70)$. Misclassification may bias the results towards the null hypothesis. Consequently, the reduction we found in the all-cause mortality risk may have been more evident.
We cannot completely exclude the influence of residual confounders. It is well known that 'healthy' behaviours occur in clusters. For example, individuals who eat a lot of whole grains will probably also have lower rates of smoking, alcohol use and obesity and higher levels of physical exercise than those who do not eat much whole grains ${ }^{(12,52)}$. Healthy lifestyles have been shown to reduce the risk of $\mathrm{CVD}^{(53,54)}$ and cancer. Most of the studies included in our meta-analysis did adjust for important possible confounders, although only four ${ }^{(13-16)}$ adjusted for other dietary factors. Studies have indicated that the intake of red meat and fruit and vegetable is significantly associated with the risk of total mortality ${ }^{(55,56)}$. Only one of the included studies adjusted for dietary fibre intake ${ }^{(16)}$. High levels of dietary fibre intake are associated with lower rates of total mortality ${ }^{(57)}$. A confounding effect of dietary fibre, red meat, fruit and vegetable intake on mortality is therefore possible. Intermediate end points such as DM, hypertension and hypercholesterolaemia that occurred during follow-up could have led to individuals changing their diets, which could have confounded the associations between diet and outcome. Reassuringly, when we restricted the analysis to studies that were adjusted for intermediate variables, the magnitude of the associations in the subgroups was similar to those overall ${ }^{(36)}$.

While the number of mortality events was large, our data came only from the USA. It will be desirable to conduct researches in other populations, particularly in oriental ones.

Finally, Egger's test did provide evidence of publication bias for the association with total mortality risk; however, the results obtained from Begg' tests did not provide evidence for such bias. Furthermore, the trim-and-fill method showed that no additional risk estimates were required to balance the funnel plot.

In conclusion, our meta-analysis of prospective cohort studies found that a higher intake of whole grains is associated with a reduced risk of all-cause and CVD-specific mortality. Further well-designed studies, including clinical trials and in different population, are required to confirm our findings.

\section{Acknowledgements}

This research received no specific grant from any funding agency, commercial or not-for-profit sectors.

H. W., Z. G. and X. L. designed the study; H. W., Z. G. and R. L. conducted the search and data extraction; H. W., Z. G. and Z. L. collected the data; Z. L., H. H. and X. L. analysed the data and wrote the manuscript. All authors read and approved the final content.

There are no conflicts of interest.

\section{Supplementary material}

For supplementary material/s referred to in this article, please visit http://dx.doi.org/doi:10.1017/S0007114516001975

\section{References}

1. United States Department of Agriculture (2000) Nutrition and Your Health: Dietary Guidelines for Americans, 5th ed. Washington, DC: US Department of Agriculture, US Department of Health and Human Services. 
2. Price RK, Wallace JM, Hamill LL, et al. (2012) Evaluation of the effect of wheat aleurone-rich foods on markers of antioxidant status, inflammation and endothelial function in apparently healthy men and women. Br J Nutr 108, 1644-1651.

3. Goletzke J, Buyken AE, Joslowski G, et al. (2014) Increased intake of carbohydrates from sources with a higher glycemic index and lower consumption of whole grains during puberty are prospectively associated with higher IL- 6 concentrations in younger adulthood among healthy individuals. J Nutr 144 1586-1593.

4. Liu S, Willett WC, Manson JE, et al. (2003) Relation between changes in intakes of dietary fiber and grain products and changes in weight and development of obesity among middle-aged women. Am J Clin Nutr 78, 920-927.

5. Steffen LM, Jacobs DR Jr, Murtaugh MA, et al. (2003) Whole grain intake is associated with lower body mass and greater insulin sensitivity among adolescents. Am J Epidemiol 158, 243-250.

6. Aune D, Norat T, Romundstad P, et al. (2013) Whole grain and refined grain consumption and the risk of type 2 diabetes: a systematic review and dose-response meta-analysis of cohort studies. Eur J Epidemiol 28, 845-858.

7. Parker ED, Liu S, Van Horn L, et al. (2013) The association of whole grain consumption with incident type 2 diabetes: the women's health initiative observational study. Ann Epidemiol 23, 321-327.

8. von Ruesten A, Feller S, Bergmann MM, et al. (2013) Diet and risk of chronic diseases: results from the first 8 years of followup in the EPIC-Potsdam study. Eur J Clin Nutr 67, 412-419.

9. Knudsen MD, Kyro C, Olsen A, et al. (2014) Self-reported whole-grain intake and plasma alkylresorcinol concentrations in combination in relation to the incidence of colorectal cancer. Am J Epidemiol 179, 1188-1196.

10. Lozano R, Naghavi M, Foreman K, et al. (2012) Global and regional mortality from 235 causes of death for 20 age groups in 1990 and 2010: a systematic analysis for the Global Burden of disease study 2010. Lancet 380, 2095-2128.

11. Liu S, Sesso HD, Manson JE, et al. (2003) Is intake of breakfast cereals related to total and cause-specific mortality in men? Am J Clin Nutr 77, 594-599.

12. Steffen LM, Jacobs DR Jr, Stevens J, et al. (2003) Associations of whole-grain, refined-grain, and fruit and vegetable consumption with risks of all-cause mortality and incident coronary artery disease and ischemic stroke: the Atherosclerosis Risk in Communities (ARIC) Study. Am J Clin Nutr 78, 383-390.

13. Jacobs DR Jr, Andersen LF \& Blomhoff R (2007) Whole-grain consumption is associated with a reduced risk of noncardiovascular, noncancer death attributed to inflammatory diseases in the Iowa Women's Health Study. Am J Clin Nutr 85, 1606-1614

14. Wu H, Flint AJ, Qi Q, et al. (2015) Association between dietary whole grain intake and risk of mortality: two large prospective studies in US men and women. JAMA Intern Med 175, 373-384.

15. Boggs DA, Ban Y, Palmer JR, et al. (2015) Higher diet quality is inversely associated with mortality in AfricanAmerican women. J Nutr 145, 547-554.

16. Huang T, Xu M, Lee A, et al. (2015) Consumption of whole grains and cereal fiber and total and cause-specific mortality: prospective analysis of 367,442 individuals. BMC Med 13, 59.

17. Sahyoun NR, Jacques PF, Zhang XL, et al. (2006) Whole-grain intake is inversely associated with the metabolic syndrome and mortality in older adults. Am J Clin Nutr 83, 124-131.

18. Jacobs DR Jr, Meyer HE \& Solvoll K (2001) Reduced mortality among whole grain bread eaters in men and women in the Norwegian County Study. Eur J Clin Nutr 55, 137-143.
19. Buil-Cosiales P, Zazpe I, Toledo E, et al. (2014) Fiber intake and all-cause mortality in the Prevencion con Dieta Mediterranea (PREDIMED) study. Am J Clin Nutr 100, 1498-1507.

20. Johnsen NF, Frederiksen K, Christensen J, et al. (2015) Wholegrain products and whole-grain types are associated with lower all-cause and cause-specific mortality in the Scandinavian HELGA cohort. Br J Nutr 114, 608-623.

21. Moher D, Liberati A, Tetzlaff J, et al. (2009) Preferred reporting items for systematic reviews and meta-analyses: the PRISMA statement. BMJ 339, b2535.

22. DerSimonian R \& Laird N (1986) Meta-analysis in clinical trials. Control Clin Trials 7, 177-188.

23. Begg CB \& Mazumdar M (1994) Operating characteristics of a rank correlation test for publication bias. Biometrics $\mathbf{5 0}$, 1088-1101.

24. Egger M, Davey Smith G, Schneider M, et al. (1997) Bias in meta-analysis detected by a simple, graphical test. BMJ $\mathbf{3 1 5}$, 629-634.

25. Duval S \& Tweedie R (2000) Trim and fill: a simple funnel-plot-based method of testing and adjusting for publication bias in meta-analysis. Biometrics 56, 455-463.

26. Higgins JP, Thompson SG, Deeks JJ, et al. (2003) Measuring inconsistency in meta-analyses. BMJ 327, 557-560.

27. Aune D, Chan DS, Lau R, et al. (2011) Dietary fibre, whole grains, and risk of colorectal cancer: systematic review and dose-response meta-analysis of prospective studies. BMJ 343, d6617.

28. Greenland S \& Longnecker MP (1992) Methods for trend estimation from summarized dose-response data, with applications to meta-analysis. Am J Epidemiol 135, 1301-1309.

29. Orsini N BR \& Greenland S (2006) Generalized least squares for trend estimation of summarized dose-response data. Stata J 6, 40-57.

30. Royston P (2000) A strategy for modelling the effect of a continuous covariate in medicine and epidemiology. Stat Med 19, $1831-1847$.

31. Flint AJ, Hu FB, Glynn RJ, et al. (2009) Whole grains and incident hypertension in men. Am J Clin Nutr 90, 493-498.

32. Wang L, Gaziano JM, Liu S, et al. (2007) Whole- and refined-grain intakes and the risk of hypertension in women. Am J Clin Nutr 86, 472-479.

33. Bazzano LA, Song Y, Bubes V, et al. (2005) Dietary intake of whole and refined grain breakfast cereals and weight gain in men. Obes Res 13, 1952-1960.

34. Koh-Banerjee P, Franz M, Sampson L, et al. (2004) Changes in whole-grain, bran, and cereal fiber consumption in relation to 8-y weight gain among men. Am J Clin Nutr 80, 1237-1245.

35. Magnusdottir OK, Landberg R, Gunnarsdottir I, et al. (2014) Whole grain rye intake, reflected by a biomarker, is associated with favorable blood lipid outcomes in subjects with the metabolic syndrome - a randomized study. PLOS ONE 9 , e110827.

36. Ye EQ, Chacko SA, Chou EL, et al. (2012) Greater whole-grain intake is associated with lower risk of type 2 diabetes, cardiovascular disease, and weight gain. J Nutr 142, 1304-1313.

37. Sun Q, Spiegelman D, van Dam RM, et al. (2010) White rice, brown rice, and risk of type 2 diabetes in US men and women. Arch Intern Med 170, 961-969.

38. Johansson DP, Lee I, Riserus U, et al. (2015) Effects of unfermented and fermented whole grain rye crisp breads served as part of a standardized breakfast, on appetite and postprandial glucose and insulin responses: a randomized cross-over trial. PLOS ONE 10, e0122241.

39. Ortiz-Martinez M, Winkler R \& Garcia-Lara S (2014) Preventive and therapeutic potential of peptides from cereals against cancer. J Proteomics 111, 165-183. 
40. Aune D, Chan DS, Lau R, et al. (2013) Dietary fibre, whole grains, and risk of colorectal cancer: systematic review and doseresponse meta-analysis of prospective studies. BMJ 3433, d6617.

41. Aarestrup J, Kyro C, Christensen J, et al. (2012) Whole grain, dietary fiber, and incidence of endometrial cancer in a Danish cohort study. Nutr Cancer 64, 1160-1168.

42. Agurs-Collins T, Rosenberg L, Makambi K, et al. (2009) Dietary patterns and breast cancer risk in women participating in the Black Women's Health Study. Am J Clin Nutr 90, 621-628.

43. Nicodemus KK \& Jacobs DR Jr (2001) Folsom AR. whole and refined grain intake and risk of incident postmenopausal breast cancer (United States). Cancer Causes Control 12, 917-925.

44. Kokubo Y, Iso H, Saito I, et al. (2011) Dietary fiber intake and risk of cardiovascular disease in the Japanese population: the Japan Public Health Center-based study cohort. Eur J Clin Nutr 65, 1233-1241.

45. Ben Q, Sun Y, Chai R, et al. (2014) Dietary fiber intake reduces risk for colorectal adenoma: a meta-analysis. Gastroenterology 146, 689-699.

46. Aune D, Chan DS, Greenwood DC, et al. (2012) Dietary fiber and breast cancer risk: a systematic review and meta-analysis of prospective studies. Ann Oncol 23, 1394-1402.

47. Jakobsdottir G, Nilsson U, Blanco N, et al. (2014) Effects of soluble and insoluble fractions from bilberries, black currants, and raspberries on short-chain fatty acid formation, anthocyanin excretion, and cholesterol in rats. J Agric Food Chem 62, 4359-4368.

48. Young GP, Hu Y, Le Leu RK, et al. (2005) Dietary fibre and colorectal cancer: a model for environment - gene interactions. Mol Nutr Food Res 49, 571-584.
49. Aburto NJ, Hanson S, Gutierrez H, et al. (2013) Effect of increased potassium intake on cardiovascular risk factors and disease: systematic review and meta-analyses. BMJ 346, f1378.

50. Nie ZL, Wang ZM, Zhou B, et al. (2013) Magnesium intake and incidence of stroke: meta-analysis of cohort studies. Nutr Metab Cardiovasc Dis 23, 169-176.

51. Muraki I, Wu H, Imamura F, et al. (2015) Rice consumption and risk of cardiovascular disease: results from a pooled analysis of 3 U.S. cohorts. Am J Clin Nutr 101, 164-172.

52. Liu S, Manson JE, Stampfer MJ, et al. (2000) Whole grain consumption and risk of ischemic stroke in women: a prospective study. JAMA 284, 1534-1540.

53. Peng M, Wu S, Jiang X, et al. (2013) Long-term alcohol consumption is an independent risk factor of hypertension development in northern China: evidence from Kailuan study. I Hypertens 31, 2342-2347.

54. Goldstein LB (2010) Physical activity and the risk of stroke. Expert Rev Neurother 10, 1263-1265.

55. Wang X, Ouyang Y, Liu J, et al. (2014) Fruit and vegetable consumption and mortality from all causes, cardiovascular disease, and cancer: systematic review and dose-response meta-analysis of prospective cohort studies. BMJ 349, g4490.

56. Abete I, Romaguera D, Vieira AR, et al. (2014) Association between total, processed, red and white meat consumption and all-cause, CVD and IHD mortality: a meta-analysis of cohort studies. Br J Nutr 112, 762-775.

57. Kim Y \& Je Y (2014) Dietary fiber intake and total mortality: a meta-analysis of prospective cohort studies. Am J Epidemiol 180, $565-573$. 